\title{
Radiopharmaceutical Chemistry of Targeted Radiotherapeutics, Part 3: $\alpha$-Particle-Induced Radiolytic Effects on the Chemical Behavior of ${ }^{211} \mathrm{At}$
}

\author{
Oscar R. Pozzi and Michael R. Zalutsky
}

Department of Radiology, Duke University Medical Center, Durham, North Carolina

Two characteristics of $\alpha$-particles that enhance their potential for targeted radiotherapy are their high energy and approximately cellular range. Unfortunately, these properties also can have negative consequences, confounding the production of clinically relevant levels of radiopharmaceutical because of radiolytic effects. The purpose of this study was to evaluate the effect of radiation dose on the astatine species present before initiation of a labeling reaction and the potential role of these molecules in the efficiency of $\mathrm{N}$-succinimidyl 3-211At-astatobenzoate (SAB) synthesis. The ranges of radiation dose evaluated were selected to reflect those that might be encountered in SAB synthesis for the preparation of clinical doses of ${ }^{211}$ At-labeled radiopharmaceuticals. Methods: The distribution of astatine species present in methanol, and the yields for the synthesis of SAB from $\mathrm{N}$-succinimidyl 3 -(tri- $n$-butylstannyl)benzoate as a function of radiation dose, were determined by high-performance liquid chromatography. Radiation doses in the range of 500-12,000 Gy were evaluated using different ${ }^{211}$ At time-activity combinations, and the effect of acetic acid, a normal component of astatodestannylation reactions, also was studied. Finally, the effect of the reducing agent sodium sulfite also was evaluated to characterize the nature of the species produced by radiolysis. Results: At radiation doses below 1,000 Gy, high-performance liquid chromatography analysis indicated that more than $90 \%$ of the ${ }^{211}$ At was present in methanol as a single species, At(1), whereas at higher doses, a second peak, At(2), emerged. At(1) decreased and At(2) increased in a radiation dose-dependent fashion, with At(2) becoming the predominant species at about 3,000 Gy. At(2) was identified as a reduced form of astatine, presumably astatide, which could not be efficiently oxidized to a species suitable for electrophilic astatination. In methanol/acetic acid, more than $95 \%$ of the astatine was present as At(2) even at doses below 1,400 Gy. Conclusion: The emergence of a reduced form of astatine, At(2), at higher radiation doses is consistent with the decline in SAB yields observed under these conditions. Alteration of the chemical form of the astatine by radiolysis could account for the declining yields noted in the preparation of clinical-level ${ }^{211}$ At-labeled radiopharmaceuticals and when the labeling chemistry is initiated hours after ${ }^{211}$ At production.

Received Nov. 29, 2006; revision accepted Mar. 23, 2007.

For correspondence or reprints contact: Michael R. Zalutsky, PhD, Department of Radiology, Duke University Medical Center, Box 3808, Durham, NC 27710.

E-mail: zalut001@mc.duke.edu

COPYRIGHT @ 2007 by the Society of Nuclear Medicine, Inc.
Key Words: ${ }^{211} \mathrm{At}$; $\alpha$-particles; radionuclide therapy; radiolysis J Nucl Med 2007; 48:1190-1196 DOI: 10.2967/jnumed.106.038505

$\mathbf{T}$ argeted $\alpha$-particle therapy is an appealing approach for therapeutic molecular medicine because the pathlength of $\alpha$-particles approximates cellular dimensions. This offers the attractive possibility of selectively eliminating malignant cell populations by exploiting their expression, either native or induced, of specific molecular targets. The potential for minimizing collateral damage to surrounding tissues coupled with high cytotoxicity are additional characteristics of $\alpha$-particles that have provided a further rationale for the development of targeted $\alpha$-particle therapy. Translational efforts in this arena currently include the targeting of tumor vasculature, blood-borne tumors, and compartmentally spread cancers and the sterilization of residual tumor tissue after surgery (1-4).

Much of this work has focused on ${ }^{211} \mathrm{At}$, a radiohalogen with a 7.2-h half-life, because this radionuclide has many attractive features for targeted radiotherapy (5). These include the association of $\alpha$-particle emission with $100 \%$ of ${ }^{211}$ At decays, no long-lived $\alpha$-particle-emitting daughters, a half-life compatible with a variety of molecular carriers, and a potentially diverse chemistry. These features have led to the investigation of a wide variety of ${ }^{211}$ At-labeled radiotherapeutics, including peptides, proteins, thymidine, and metaiodobenzylguanidine analogs, as well as other small molecules (6-11). However, because of the scarcity of cyclotrons equipped with $25-$ to $30-\mathrm{MeV} \alpha$-particle beams-a requirement for efficient ${ }^{211} \mathrm{At}$ production-radionuclide availability is a major impediment to the clinical translation of these promising radiopharmaceuticals.

The fact that ${ }^{211}$ At-labeled therapeutics would by necessity generally be used at locations distant from a ${ }^{211} \mathrm{At}$ production site presents a major challenge to the chemist because, under these circumstances, the astatine activity 
frequently arrives in a form that results in unacceptable labeling yields. The diminishing efficiency of electrophilic astatination reactions with the passage of time is well known, even when ${ }^{211}$ At is used at its site of production. These difficulties likely relate to the cumulative effect of ${ }^{211}$ At $\alpha$-particles depositing large amounts of decay energy in a highly localized manner. The radiolytic consequences of $\alpha$-particle decay also can interfere with the preparation of clinically relevant levels of ${ }^{211}$ At-labeled radiopharmaceuticals such as $\mathrm{N}$-succinimidyl $3-{ }^{211} \mathrm{At}$-astatobenzoate (SAB), which is used for labeling monoclonal antibodies (mAbs) (12).

In previous publications, we have investigated the effects of increasing $\alpha$-particle radiation dose on the tin precursor required for $\mathrm{SAB}$ synthesis (13) and the nature of the labeled products that were generated (14). The goal of the present study was to evaluate the effect of radiation dose on the astatine species present before initiation of the labeling reaction and the potential role of these molecules on labeling efficiency, using SAB as the model compound. Astatine is the heaviest of the halogens and has the lowest ionization energy of the group $(920 \mathrm{~kJ} / \mathrm{mol})$. The element exists in multiple oxidation states- $\mathrm{At}^{-}, \mathrm{At}^{+}, \mathrm{At}^{+3}\left(\mathrm{AtO}_{2}^{-}\right)$, and $\mathrm{At}^{+5}\left(\mathrm{AtO}_{3}^{-}\right)$- and has an electronic configuration that makes it very reactive (15). For these reasons, we hypothesized that the chemical nature of ${ }^{211}$ At can be modified by interaction with highly reactive, radiolytically generated species coming from the solvent. Because previous work showed that methanol is a better solvent than benzene or chloroform for electrophilic astatination of organometallic derivatives at elevated radiation dose levels $(13,14)$, all experiments described herein used methanol as the solvent.

\section{MATERIALS AND METHODS}

\section{General}

Methanol was obtained from Aldrich and was of anhydrous 99.8\% grade (Sure/Seal bottles [Sigma-Aldrich] with $<0.002 \%$ water and $<0.0003 \%$ evaporation residue). All other solvents were of reagent grade or better and were used as purchased. The $\mathrm{Na}^{131} \mathrm{I}$ was obtained from Nordion Canada (NEZ035H; $65 \mathrm{GBq} / \mathrm{mL}$; specific activity, $679 \mathrm{MBq} / \mathrm{mg}$ ). The high-performance liquid chromatography (HPLC) analyses were performed on a System Gold HPLC system (Beckman) equipped with a diode array detector and a radioisotope detector. For reversed-phase chromatography, an Xterra (Waters) $4.6 \times 250 \mathrm{~mm}(10-\mu \mathrm{m})$ column was used. The elution for reverse-phase chromatography was made with a gradient of solvent B (acetonitrile/acetic acid $0.1 \%$ ) in solvent A (acetonitrile/water/acetic acid [5/95/0.1]) held at $28 \%$ of B for $15 \mathrm{~min}$, followed by a $28 \%$-to- $100 \%$ linear gradient of B over $20 \mathrm{~min}$, followed by $100 \%$ solvent $\mathrm{B}$ for the remainder of the HPLC run. The flow rate was $1 \mathrm{~mL} / \mathrm{min}$.

Electrophoresis was measured using a PowerPac Basic power supply (Biorad) run at $250 \mathrm{~V}$ and $50 \mathrm{~mA}$ for $15 \mathrm{~min}$. Grade 1 glass fiber strips $(1 \times 20 \mathrm{~cm}$; Whatman $)$ were run in $0.05 \mathrm{M}$ phosphate buffer, $\mathrm{pH}$ 7. The strips were analyzed immediately after the run using an imaging scanner (model 200; Bioscan).
SAB synthesis experiments were performed by astatodestannylation of $N$-succinimidyl 3-(tri- $n$-butylstannyl)benzoate (BuSTB), which was prepared as described previously $(16,17)$. The purity of the tin precursor was confirmed before each set of experiments by thin-layer chromatography.

\section{Production of ${ }^{211}$ At and Radiation Dose Calculations}

The ${ }^{211}$ At was produced at the Duke University Medical Center cyclotron and an internal target system (18) by bombarding natural bismuth metal targets with $28.0-\mathrm{MeV} \alpha$-particles using the ${ }^{209} \mathrm{Bi}(\alpha, 2 \mathrm{n})^{211}$ At reaction. The dry distillation system for isolation of the ${ }^{211}$ At from the bismuth cyclotron target was a variation of a system described previously $(19,20)$. The current distillation device uses a polyetheretherketone (PEEK) capillary loop (1.6$\mathrm{mm}\left[1 / 16^{1}\right.$-in] tubing, $1-\mathrm{mm}$ inner diameter $\times 152$-cm [5-ft] length) obtained from Upchurch Scientific instead of the glass vials that were used in the past (12) for trapping the ${ }^{211}$ At activity. One reason for this change was to delay the time at which the ${ }^{211} \mathrm{At}$ first was exposed to the solvent. In addition, this change permitted a more accurate calculation of the radiation dose received by the solvent. During the distillation, the PEEK tubing was chilled using an ethanol dry-ice bath. A gas wash-bottle trap containing a reducing agent was placed downstream but before the vacuum pump, to retain any astatine that might escape from the PEEK tubing trap. Other aspects of the distillation procedure-the rest of the device, furnace, distillation temperature, quartz still, and argon flowwere similar to those used in the past (12). At the end of the distillation, the tubing was removed from the apparatus, and both ends were covered with all-purpose laboratory film (Parafilm; Alcan Inc.) and allowed to warm to room temperature. The astatine captured in the PEEK tubing was eluted just before each experiment into a small volume of methanol, normally in the range of 300-750 $\mu \mathrm{L}$. The activity, time, and volume were recorded to calculate the radiation dose received by the solvent.

For the radiation dose calculation, all $\alpha$-particle and $\alpha$-recoil nuclei decay energy was assumed to be deposited in the solution because of the short range of these emissions $(<100 \mu \mathrm{m})$ relative to the dimensions of the reaction mixtures. Uniform distribution of the reactants in the solvent also was assumed. The absorbed dose was calculated as (12):

$$
D=A_{i}\left(1-e^{-\lambda t}\right) \frac{1}{\lambda} \frac{1}{m} \Delta_{i}
$$

where $D$ is expressed in grays, $A_{i}$ is the initial activity in megabecquerels, $\lambda$ is the decay constant for ${ }^{211} \mathrm{At}\left(\mathrm{s}^{-1}\right), t$ is the exposure time in seconds, $m$ is the mass of the solution $(\mathrm{g})$, and $\Delta_{i}$ is the mean energy emitted per nuclear transition. Based on dose contributions from $\alpha$-particles and $\alpha$-recoil nuclei, a $\Delta_{i}$ of $1.09 \times$ $10^{-3} \mathrm{~Gy} \cdot \mathrm{g} / \mathrm{MBq} \cdot \mathrm{s}$ was calculated for ${ }^{211}$ At decay (21). A density of $0.791 \mathrm{~g} / \mathrm{mL}$ was used to convert methanol volume to mass.

\section{Radiolysis Experiments and SAB Synthesis}

To study the influence of $\mathrm{pH}$, we performed the experiments both with methanol alone and with methanol adjusted to an acidic $\mathrm{pH}$ (5.0-5.5) with acetic acid $(0.67 \mathrm{~mol} / \mathrm{L})$, the conditions normally used for SAB synthesis (12). All the experiments were conducted under atmospheric conditions and at room temperature.

The radiation dose ranges evaluated herein were selected to reflect those that might be encountered in labeling SAB for the preparation of clinical doses of ${ }^{211}$ At-labeled radiopharmaceuticals. 
In a previous study, difficulties were encountered in reproducible $\mathrm{SAB}$ preparation and efficient $\mathrm{mAb}$ conjugation when $370 \mathrm{MBq}$ of ${ }^{211}$ At-labeled $\mathrm{mAb}$ were required (12). Based on previously determined yields for $\mathrm{SAB}$ synthesis and mAb conjugation, and the times required for synthesis and purification, greater than $1,500 \mathrm{MBq}$ of initial ${ }^{211}$ At activity would be needed to prepare this activity level of labeled mAb. At the volume used for SAB synthesis, a dose of 4,700 Gy would be delivered to the methanol reaction mixture (13). For this reason, experiments were performed under conditions resulting in the delivery of radiation doses both above and below this 4,700-Gy benchmark.

The activity levels of ${ }^{211}$ At used in each run ranged between about 40 and $160 \mathrm{MBq}$ and were measured using a CRC-7 dose calibrator (Capintec). Samples for HPLC analysis were taken at various times that were selected on the basis of the approximate radiation dose levels of interest in each experiment. The radiation dose deposited in the reaction vessel ranged between about 500 and 12,000 Gy, with exposure periods ranging between minutes and about $24 \mathrm{~h}$. The dose rates were kept below $1 \mathrm{~Gy} / \mathrm{s}$ to facilitate comparison with previous experiments from our database. Samples were injected onto the HPLC column immediately after they were obtained. The areas of all astatinated species were integrated, and the results were expressed as the percentage of total activity eluted from the column and were plotted as a function of radiation dose (Gy).

When the synthesis of SAB was investigated, it was prepared by adding $50 \mu \mathrm{g}$ of BuSTB in $50 \mu \mathrm{L}$ of methanol, $100 \mu \mathrm{g}$ of $N$-chlorosuccinimide (NCS) dissolved in $50 \mu \mathrm{L}$ of methanol, and $20 \mu \mathrm{L}$ of acetic acid to $0.4 \mathrm{~mL}$ of ${ }^{211} \mathrm{At} / \mathrm{methanol}$ in a reaction vial (Reacti-vial; Pierce), unless different conditions were specified. The reaction mixture was shaken for $20 \mathrm{~min}$, and $50-\mu \mathrm{L}$ aliquots were periodically removed for HPLC analysis. To study the effect of $\mathrm{pH}$, parallel reactions were performed on the same day, with and without acetic acid at the same final volume. For the experiment directed at attempting to characterize the chemical form of ${ }^{211} \mathrm{At} / \mathrm{methanol}$ under high-level radiation dose conditions, a $30-\mu \mathrm{L}$ aliquot of ${ }^{211} \mathrm{At} /$ methanol was combined with $30 \mu \mathrm{L}$ of sodium sulfite $(2 \mu \mathrm{mol})$. Sodium sulfite is a reducing agent used routinely for the generation of astatide (15) and was allowed to react for $30 \mathrm{~min}$. HPLC chromatograms were obtained for aliquots of the ${ }^{211}$ At in methanol, both before and after the addition of the sodium sulfite. Approximately equal levels of radioactivity were injected onto the HPLC column by adjusting the volume of the 2 aliquots that were analyzed.

\section{RESULTS}

The effect of radiation dose on the chemical form of astatine was evaluated using HPLC. In a typical experiment, ${ }^{211}$ At was eluted into methanol $(58 \mathrm{MBq} / 750 \mu \mathrm{L})$ and stored for $21.5 \mathrm{~h}$ at room temperature, resulting in a radiation dose of 3,630 Gy (Fig. 1). HPLC analysis indicated that essentially all ${ }^{211}$ At activity that eluted from the column (77\%-89\% of the total activity added to the column for all HPLC runs presented in this study) was found in 2 peaks eluting at 6.2 and $10.7 \mathrm{~min}$, designated hereafter as $\operatorname{At}(1)$ and $\operatorname{At}(2)$, respectively. The first peak, observed at $6.2 \mathrm{~min}$, corresponded to that observed in freshly prepared ${ }^{211} \mathrm{At} / \mathrm{methanol} \mathrm{solutions} \mathrm{and} \mathrm{those} \mathrm{exposed} \mathrm{to} \mathrm{doses} \mathrm{of} \mathrm{less}$ than 1,500 Gy.

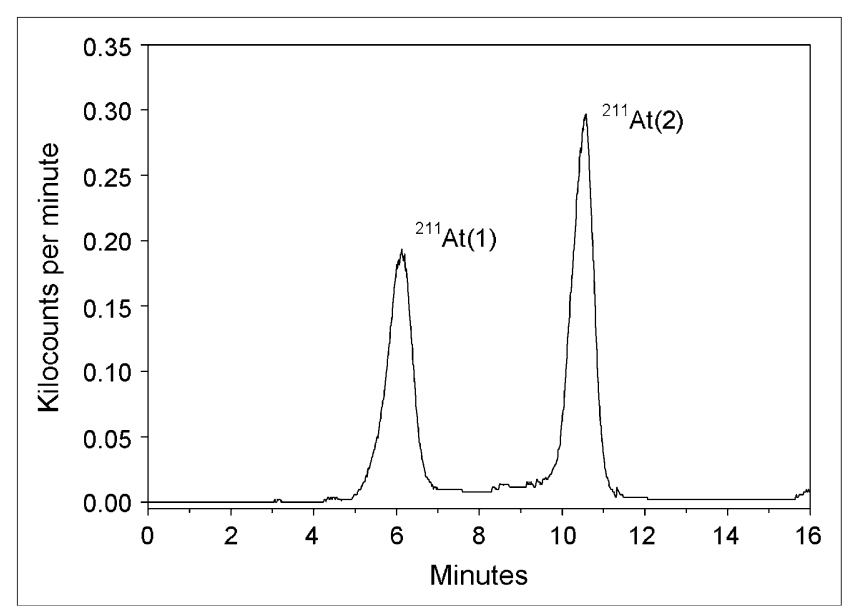

FIGURE 1. Representative reverse-phase HPLC profile of radioactive species present in methanol solution after exposure to ${ }^{211} \mathrm{At}$. In this example, radiation dose received by solution was 3,630 Gy.

The effect of radiation dose on ${ }^{211} \mathrm{At} /$ methanol solutions was further evaluated by taking aliquots at successive periods, yielding increasing calculated radiation doses. The results for an experiment performed with an initial activity concentration of $43.5 \mathrm{MBq} / 540 \mu \mathrm{L}$ are shown in Figure 2 . The distribution of eluted ${ }^{211}$ At activity between the 2 HPLC peaks clearly was dependent on the radiation dose received by the methanol, but not in a linear fashion. The contribution of $\operatorname{At}(2)$ increased gradually until about 2,200 Gy and then increased more rapidly, becoming the predominant species at radiation doses above about 3,200 Gy. Similar behavior, but with a slower increase of $\operatorname{At}(2)$ contribution, was observed in other experiments performed at higher dose rates, and experiments are currently in progress to explore potential dose rate effects in more detail.

Because acetic acid is a standard component in electrophilic astatination reactions, the ${ }^{211}$ At species generated in ${ }^{211} \mathrm{At} / \mathrm{methanol} \mathrm{solutions}$ containing acetic acid $(0.67 \mathrm{~mol} / \mathrm{L})$

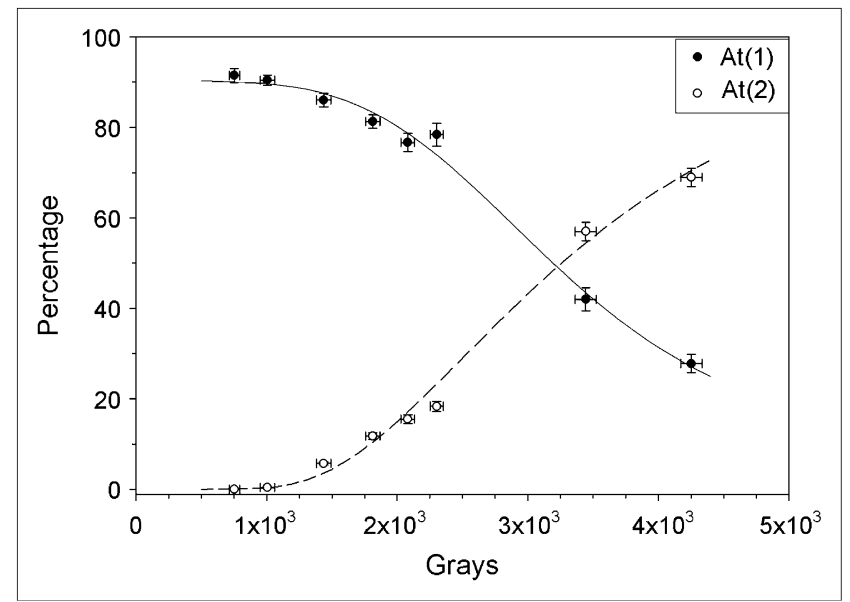

FIGURE 2. Relative amounts of At(1) and At(2), as shown in Figure 1, in methanol (without acetic acid) as function of radiation dose absorbed by solvent. 
was evaluated. At all doses investigated (1,000-20,000 Gy), at least $95 \%$ of eluted ${ }^{211}$ At activity was found in the $\operatorname{At}(2)$ form. Thus, at low radiation doses, astatine is present in different forms in methanol with or without acetic acid, whereas at higher radiation doses, $\operatorname{At}(2)$ predominates in both cases.

Based on the fact that methanol radiolysis is known to generate reducing species, particularly at an acidic $\mathrm{pH}$ (14), these observations suggested that $\operatorname{At}(2)$ is a reduced form of astatine, most likely astatide. To investigate this possibility further, ${ }^{211} \mathrm{At} /$ methanol $(160.6 \mathrm{MBq} / 460 \mu \mathrm{L})$ was treated with sodium sulfite. A small aliquot with a low level of ${ }^{211}$ At activity was used to minimize radiation dose deposition during treatment with the reducing agent. HPLC analysis was performed on an aliquot of ${ }^{211} \mathrm{At}$ in methanol obtained before and $30 \mathrm{~min}$ after sodium sulfite addition. Treatment with sulfite decreased the fraction of eluted activity in the At(1) peak from $46 \%$ of eluted activity to less than $1 \%$, whereas the contribution from the $\operatorname{At}(2)$ peak increased from $52 \%$ to $90 \%$. Addition of the reducing agent resulted in almost complete conversion of $\operatorname{At}(1)$ to $\operatorname{At}(2)$ in 5 other paired experiments performed at different initial activity concentrations, providing further evidence that $\operatorname{At}(2)$ represents a reduced form of astatine. Furthermore, analysis of $\mathrm{Na}^{131}$ I-iodide/methanol solutions under the same HPLC conditions showed that iodide eluted as a single peak at $10.2 \mathrm{~min}$, a retention time quite similar to that observed for $\operatorname{At}(2)$.

To better understand the chemical nature of the 2 species observed in the HPLC chromatograms, we analyzed ${ }^{211} \mathrm{At} /$ methanol solutions by electrophoresis. Figure 3 shows a direct comparison of the HPLC and electrophoresis profiles obtained from the same methanol solution after exposure to ${ }^{211}$ At. Two peaks with similar relative magnitudes are observed with both techniques. In the electrophoresis profile, the larger peak is found at the origin, indicating that it

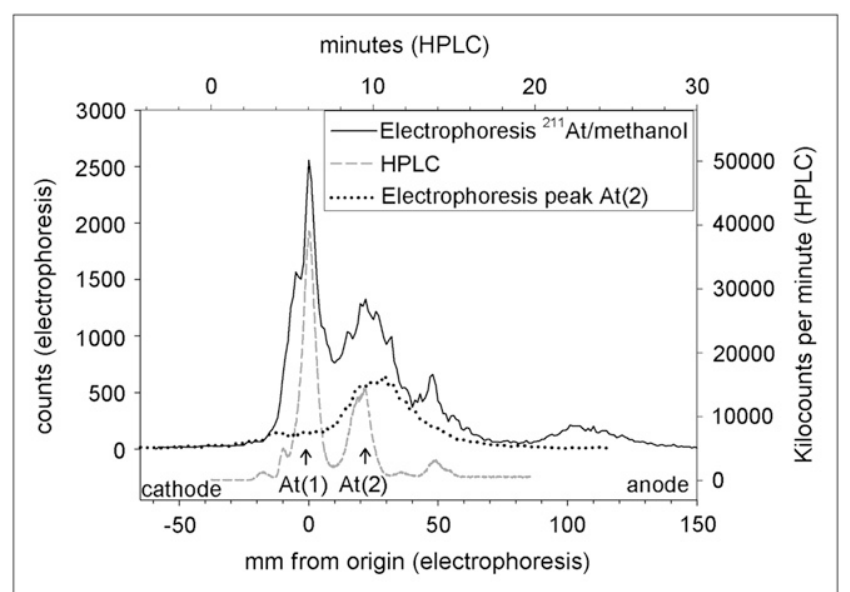

FIGURE 3. Analysis of same ${ }^{211}$ At in methanol solution by reverse-phase HPLC (dashed line) and paper electrophoresis (solid line). Electrophoretic analysis of sample of At(2) peak observed on HPLC chromatogram also was performed (dotted line). is neutral, whereas the smaller peak migrates toward the anode, indicating that it is negatively charged. A sample was obtained from the At(2) peak, isolated by HPLC, and its electrophoretic behavior was evaluated. A single species migrating toward the anode was observed, confirming that $\operatorname{At}(2)$ is a negatively charged species.

The peak corresponding to At(1) predominates in fresh ${ }^{211} \mathrm{At} /$ methanol solutions at low radiation doses, conditions under which electrophilic astatination reactions give high reaction yields. The emergence of a reduced form of astatine, At(2), at higher radiation doses could account for the decline in SAB yields under these conditions. However, a potential compensatory strategy is also suggested: treatment of $\operatorname{At}(2)$ with an oxidant such as NCS to generate a reactive electrophilic astatine species.

To investigate this possibility, we evaluated the synthesis of SAB from a ${ }^{211} \mathrm{At} /$ methanol solution in which $46 \%$ and $52 \%$ of the activity were present as $\operatorname{At}(1)$ and $\operatorname{At}(2)$, respectively, determined by HPLC, at the initiation of SAB synthesis. The ${ }^{211}$ At and methanol (2 aliquots of $200 \mu \mathrm{L}$ each) were added to 2 vials containing $80 \mu \mathrm{g}$ of BuSTB and $200 \mu \mathrm{g}$ of NCS in $100 \mu \mathrm{L}$ of methanol; 1 reaction mixture also contained acetic acid $(0.67 \mathrm{~mol} / \mathrm{L})$. The almost complete disappearance of the $\operatorname{At}(1)$ peak with concomitant $\mathrm{SAB}$ production suggests that that this astatine species is reactive toward electrophilic astatination (Fig. 4). In contrast, about half the $\operatorname{At}(2)$ generated by radiolysis before the SAB reaction was not oxidized by NCS to a species suitable for electrophilic astatination (Fig. 4A). Although a greater fraction of $\operatorname{At}(2)$ could be converted to $\mathrm{SAB}$ under acidic than under neutral reaction conditions $(\sim 50 \%$ vs. $\sim 10 \%$ ) by adding NCS to the reaction mixture, these results suggest that this strategy is not efficient for converting $\operatorname{At}(2)$ to the desired product.

Next, ${ }^{211} \mathrm{At} / \mathrm{methanol}$ was reacted with BuSTB at 4 different radiation doses at a neutral $\mathrm{pH}$ without the addition of NCS to determine whether SAB yield could be related to At(2) fraction. These conditions were selected because both NCS and acetic acid could alter the chemical forms of astatine that are present. Previously (14), we observed that $\mathrm{SAB}$ could be generated in the absence of NCS; however, this radiolysis-induced SAB decreased with increasing radiation dose and was significantly higher without acetic acid. As shown in Fig. 5, an inverse relationship existed between SAB yield and the fraction of ${ }^{211} \mathrm{At} /$ methanol present as $\operatorname{At}(2)$ at the initiation of the reaction. This finding further supports the hypothesis that, for $\mathrm{SAB}$ synthesis, At(2) is less reactive than At(1).

We next investigated the effect of acetic acid on SAB production in the presence of NCS in paired reactions using ${ }^{211} \mathrm{At} /$ methanol solutions that had received a relatively low radiation dose $(\sim 2,000 \mathrm{~Gy})$ to minimize radiolysis-mediated production of the less reactive At(2) species. Under these conditions, SAB yields were essentially identical in the presence $(73 \%)$ or absence $(74 \%)$ of acetic acid. Thus, when the radiation dose received before initiation of the 

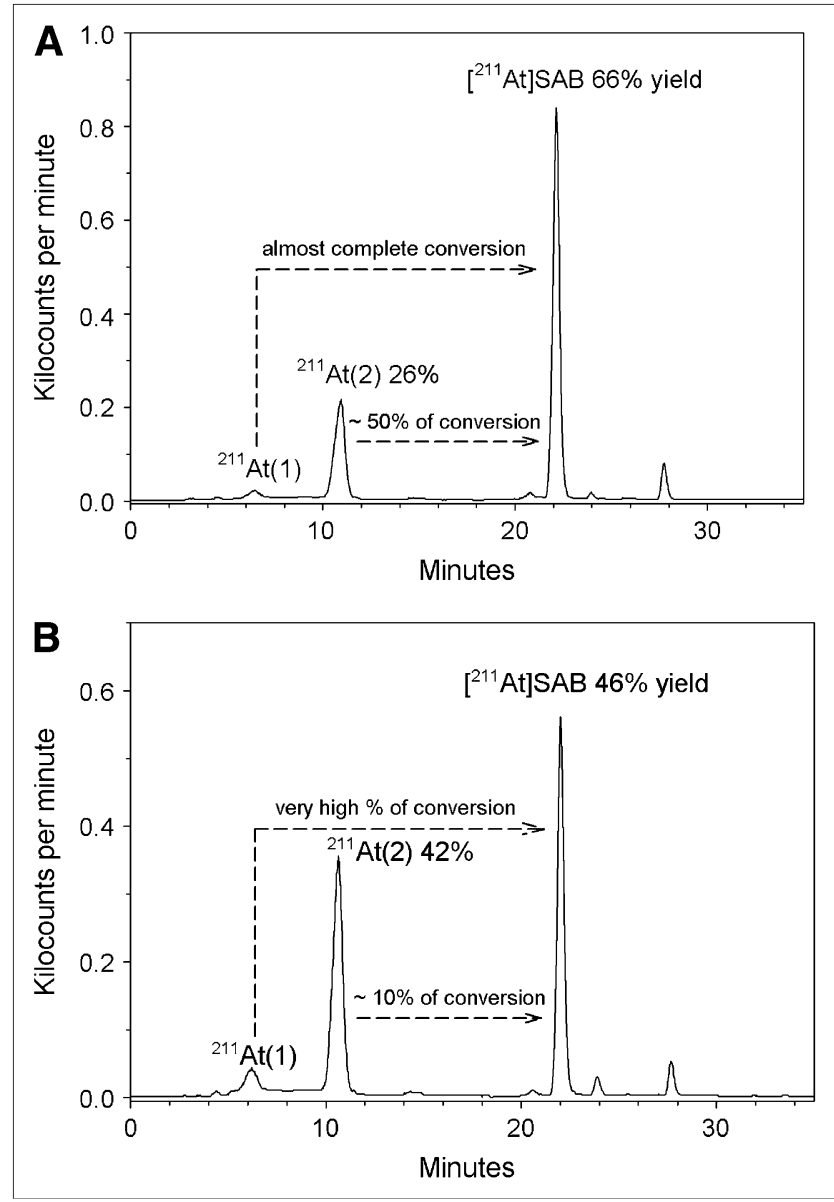

FIGURE 4. Effect of presence $(A)$ or absence $(B)$ of acetic acid $(0.67 \mathrm{~mol} / \mathrm{L})$ on synthesis of SAB from ${ }^{211} \mathrm{At} / \mathrm{methanol}$ solutions at radiation dose resulting in presence of $A t(1)$ and $A t(2)$. Arrows indicate percentage conversion of $A t(1)$ and $A t(2)$ to $S A B$, calculated by comparison to levels of $A t(1)$ and $A t(2)$ present at initiation of reaction and determined by HPLC.

labeling reaction in methanol was low, running the reaction with NCS at a lower pH did not increase yields. On the other hand, the opposite behavior was observed at high radiation doses (Fig. 4), presumably reflecting more efficient conversion of $\mathrm{At}(2)$ to $\mathrm{SAB}$ in the presence of acetic acid. This greater efficiency is in contrast to the radiolysisinduced synthesis of SAB (i.e., in the absence of oxidant), which is less efficient in the presence of acetic acid (14).

\section{DISCUSSION}

Targeted $\alpha$-particle radiotherapy is a promising approach to cancer treatment because, at least in principle, curative doses of radiation can be selectively delivered to malignant cells on the basis of their expression of oncogenically derived molecular markers. However, translation of concept to clinical practice has been slow because of many hurdles, including the need for more sophisticated dosimetric methodologies and normal-organ toxicity data, as well as limited availability of $\alpha$-emitters with appropriate characteristics

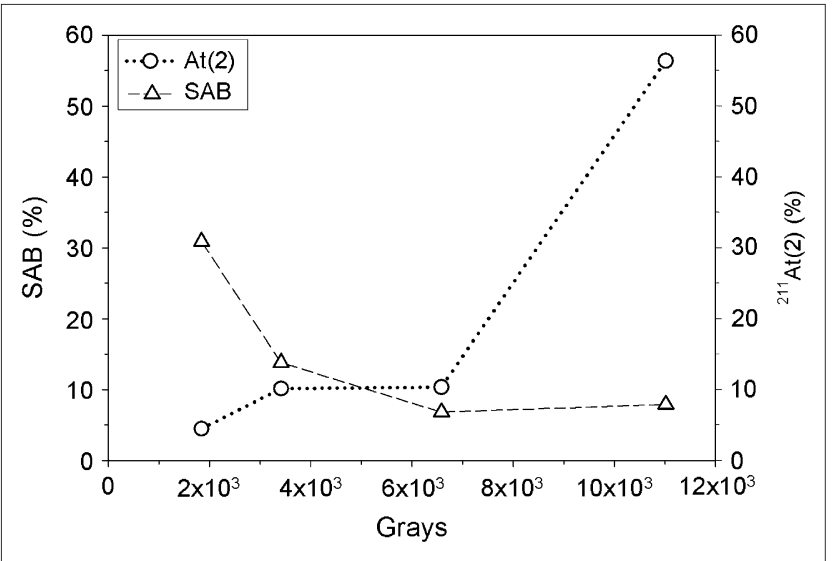

FIGURE 5. Production of SAB from reaction of BuSTB and ${ }^{211} \mathrm{At}$ in methanol by radiolysis (i.e., no oxidant or acetic acid added), compared with percentage of ${ }^{211} \mathrm{At}(2)$ present as function of radiation dose.

$(2,3,22)$. Moreover, labeling strategies must be developed that are practical and reliable at the elevated dose levels required for patient treatment.

Such methodologies were critical to bringing ${ }^{131} \mathrm{I}$ tositumomab (Bexxar; GlaxoSmithKline) and ${ }^{90}$ Y-ibiritumonab tiuxetan (Zevalin; Biogen Idec, Inc.) to the marketplace; however, achieving similar success with an $\alpha$-emitter such as ${ }^{211} \mathrm{At}$ will be an even greater challenge. Compared with these $\beta$-emitters, ${ }^{211}$ At has a half-life shorter by an order of magnitude but deposits an energy per volume greater by 2 orders of magnitude than ${ }^{90} \mathrm{Y}$ or ${ }^{131} \mathrm{I}$ (23). Because of these differences, the potential impact of radiolytic effects on ${ }^{211} \mathrm{At}$ labeling chemistry is considerably higher. Particularly for institutions distant from the site of ${ }^{211}$ At production, the radiation dose received before initiation of the labeling reaction will be considerable. Even when clinical-level ${ }^{211} \mathrm{At} \mathrm{mAb}$ labeling was performed with the radionuclide produced on site, radiation doses of up to approximately 4,000 Gy were delivered in the 5-40 min before SAB synthesis began (12).

The rapidly declining SAB yield with increasing radiation dose under conditions in which the tin precursor remained largely intact led us to hypothesize that this behavior was due to radiation-induced changes in the chemical form of astatine (14). The results of the current study are consistent with this hypothesis.

In Figure 6, the fraction of activity that would be present as $\mathrm{At}(1)$ at the beginning of a hypothetical reaction (Fig. 2) is compared with SAB yields determined previously (14) as a function of the radiation dose received by the ${ }^{211} \mathrm{At} /$ methanol solution before initiation of the destannylation reaction. Although this comparison must be done with caution, it is worth noting the at least qualitatively similar decline in At(1) fraction and SAB yield as a function of radiation dose. This finding suggests that $\operatorname{At}(2)$ is in a chemical form from which $\mathrm{At}^{+}$, the species generally considered to be required for electrophilic astatodestannylaton, cannot be efficiently generated. 


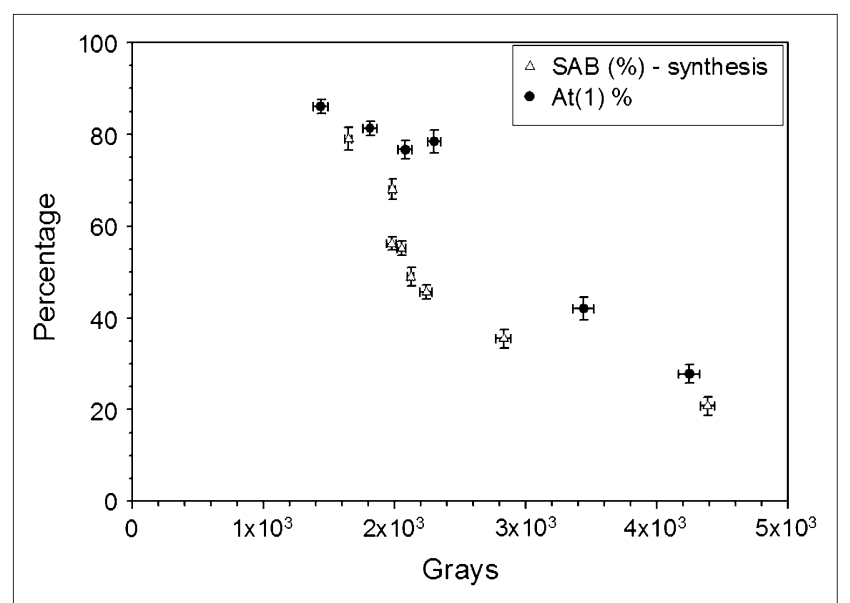

FIGURE 6. Relative amounts of ${ }^{211} \mathrm{At}(1)$ in pure methanol (data from Fig. 2) vs. yield of $S A B$ synthesis made in methanol with oxidant and acetic acid (0.67 mol/L) (data from (14)).

Unfortunately, definitive chemical identification of At(1) and At(2) could not be done because the lack of a stable astatine isotope limits the use of standard analytic techniques. Because iodine is the element most similar to astatine, its chemistry might shed some light on the behavior of astatine. Of potential relevance to the current work is a theoretic study of complex formation of iodine with methanol (24). These complexes are formed through acid-base types of interactions in which iodine functions as the acid or electron acceptor. Methanol contains relatively mobile protons, making acid-base types of interactions and the formation of complexes of this kind possible. If astatine also interacted with methanol in a similar fashion, the peak At(1) could be a complex of this kind. However, we cannot be certain at this time even whether this peak corresponds to a single astatine species.

In contrast to low-linear-energy transfer radiation, such as $\beta$-emitters, the dominant mechanism for $\alpha$-particlemediated radiolysis is spur overlap, a consequence of their densely ionizing nature $(25,26)$. As the number of $\alpha$-decays per unit time (i.e., dose rate) increases, the probability of intertrack overlap increases until, at very high dose rates, a nearly homogeneous environment is created (27). This decreases the yield of radicals and favors the formation of molecular products within the spur (26). An important consequence of methanol radiolysis in that regard is the generation of hydrogen and formaldehyde, which are reducing species (28). These molecules can then interact with astatine atoms that have not decayed, potentially altering their oxidation state and chemical form. Because of its carrierfree nature, when ${ }^{211} \mathrm{At}$ is isolated from the cyclotron target in the absence of oxidant, the number of moles of reducing species produced from methanol radiolysis could exceed those of astatine by several orders of magnitude, even at relatively low radiation doses. This could lead to the generation of reduced forms of astatine, which are not suitable for electrophilic astatination. Our results demonstrating an increasing fraction of $\operatorname{At}(2)$ with increasing radiation dose are consistent with this hypothesis.

We hypothesize that $\operatorname{At}(2)$ is astatide for several reasons. First, At(1) is nearly completely converted to At(2) by treatment with the reducing agent sodium sulfite. Second, the retention time of $\operatorname{At}(2)$ was nearly identical to that of sodium ${ }^{131}$ I-iodide. And third, At(2) was demonstrated to be an anionic species by electrophoresis, whereas At(1) was shown to be a neutral species. It is worth noting that the yields of SAB generally are higher when astatine is present in an organic solvent, where it is presumed to be in the zero valence state (29-31). Thus, it appears that radiolysis of methanol could have 2 deleterious effects on electrophilic astatination reactions-consumption of the oxidant added to the reaction to create the electrophilic astatine species and reduction of the ${ }^{211} \mathrm{At}$, making generation of electrophilic astatine more difficult.

The reactions occurring during methanol radiolysis provide a rationale for the dose- and $\mathrm{pH}$-dependent increase in At(2) production. In methanol, radiation renders the spur more acidic than the bulk because of the formation of $\mathrm{CH}_{3} \mathrm{OH}^{2+}$, and acidic $\mathrm{pH}$ increases the production of reducing species in methanol (28). Thus, as the radiation dose increases, the spur becomes more acidic, which, in turn, increases generation of reducing species. We speculate that the sequence "more acidic spur $\rightarrow$ more reducing species" results in increased $\operatorname{At}(2)$ production, which will continue increasing incrementally as the spur overlap increases. Consistent with this speculation is the observation of increased production of $\operatorname{At}(2)$ at lower radiation doses in the presence, compared with the absence, of acetic acid.

Alteration of the $\mathrm{pH}$ at a microscopic level could also account for the observation that the addition of acetic acid did not increase SAB yield when a fresh ${ }^{211} \mathrm{At} /$ methanol solution was used. An acidic $\mathrm{pH}$ is well known to be required for efficient electrophilic halogenation (30). The radiation doses received during these $\mathrm{SAB}$ syntheses were only about $20 \%$ of those received during a clinical therapylevel SAB synthesis. We speculate that even this radiation dose might have been sufficient to create a microscopiclevel acidic $\mathrm{pH}$ environment, obviating the addition of acetic acid to the SAB synthesis reaction mixture. Recently, the addition of acetic acid was reported to have no effect on the synthesis of analogous ${ }^{211}$ At-labeled $N$-succinimidyl benzoates from their corresponding tin precursors at similar activity levels (32).

\section{CONCLUSION}

${ }^{211} \mathrm{At}$ is one of the most promising $\alpha$-particle emitters for targeted radiotherapy. However, the radiolysis effects induced by these high-linear-energy particles can interfere with labeling chemistry either when performed at clinically relevant activity levels or when the reaction is initiated hours after ${ }^{211}$ At production. Previous studies using the wellknown protein conjugation agent $\mathrm{SAB}$ revealed 2 problems: a competitive reaction of radiolysis-generated species with 
the tin precursor (13) and a reaction of the ${ }^{211}$ At with the solvent itself at high radiation doses (14). Our results presented here demonstrate for, what is to our knowledge, the first time another significant impediment to successful astatine chemistry: alteration of the chemical forms of ${ }^{211}$ At with increasing radiation doses from forms suitable for electrophilic labeling reactions to forms that are not. We show that in methanol (the optimal solvent identified to date for therapy-level astatodestannylation), astatine is present at low radiation doses in a form from which At + can readily be generated, perhaps stabilized in a complex with methanol. With increasing deposition of radiation dose to the solvent, conversion to a reduced species, probably astatide, occurs, accounting for the decline in labeling yields at elevated radiation doses. More solvents need to be investigated to gain a better understanding of radiolytic effects on reactions of this type. In conclusion, the results of this study underscore the potential importance of radiolysismediated effects on the chemistry of $\alpha$-particle-emitting radiopharmaceuticals and the need to evaluate labeling chemistry at the high radiation doses required for clinical use.

\section{ACKNOWLEDGMENTS}

We thank Marc Hens and Kevin Alston for excellent technical assistance. This work was supported in part by grants CA42324 and NS20023 from the National Institutes of Health, grant DE-FG02-05ER63963 from the U.S. Department of Energy, and a grant from the Pediatric Brain Tumor Foundation.

\section{REFERENCES}

1. Mulford DA, Scheinberg DA, Jurcic JG. The promise of targeted $\alpha$-particle therapy. J Nucl Med. 2005;46(suppl):199S-204S.

2. Welch M. Potential and pitfalls of therapy with $\alpha$-particles. J Nucl Med. 2005; 46:1254-1255.

3. Zalutsky MR. Targeted $\alpha$-particle therapy of microscopic disease: providing a further rationale for clinical investigation. J Nucl Med. 2006;47:1238-1240.

4. Couturier O, Supiot S, Degraef-Mougin M, et al. Cancer radioimmuno-therapy with alpha-emitting nuclides. Eur J Nucl Med Mol Imaging. 2005;32:601-614.

5. Zalutsky MR, Vaidyanathan G. Astatine-211-labeled radiotherapeutics: an emerging approach to targeted alpha particle therapy. Curr Pharm Des. 2000;6:14331455 .

6. Elgqvist J, Andersson H, Bäck T, et al. $\alpha$-Radioimmunotherapy of intraperitoneally growing OVCAR-3 tumors of variable dimensions: outcome related to measured tumor size and mean absorbed dose. J Nucl Med. 2006;47:13421350 .

7. Sundberg AL, Almqvist Y, Orlova A, et al. Combined effect of gefitinib ('Iressa', ZD1839) and targeted radiotherapy with ${ }^{211}$ At-EGF. Eur J Nucl Med Mol Imaging. 2003;30:1348-1356.

8. Wilbur DS, Chyan MK, Hamlin DK, et al. Reagents for astatination of biomolecules: comparison of the in vivo distribution and stability of some radioiodinated/astatinated benzamidyl and nido-carboranyl compounds. Bioconjug Chem. 2004;15:203-223.
9. Murud KM, Larsen RH, Bruland OS, Hoff P. Influence of pretreatment with 3-amino-1-hydroxypropylidene-1,1-bisphosphonate (APB) on organ uptake of ${ }^{211}$ At and ${ }^{125}$ I-labeled amidobisphosphonates in mice. Nucl Med Biol. 1999;26: 791-794.

10. Demartis S, Tarli L, Borsi L, Zardi L, Neri D. Selective targeting of tumour neovasculature by a radiohalogenated human antibody fragment specific for the ED-B domain of fibronectin. Eur J Nucl Med. 2001;28:534-539.

11. Walicka MA, Vaidyanathan G, Zalutsky MR, Adelstein SJ, Kassis AI. Survival and DNA damage in Chinese hamster V79 cells exposed to alpha particles emitted by DNA-incorporated astatine-211. Radiat Res. 1998;150:263-268.

12. Zalutsky MR, Zhao X-G, Alston KL, Bigner DD. High-level production of $\alpha$-particle-emitting ${ }^{211} \mathrm{At}$ and preparation of ${ }^{211}$ At-labeled antibodies for clinical use. J Nucl Med. 2001;42:1508-1515.

13. Pozzi OR, Zalutsky MR. Radiopharmaceutical chemistry of targeted radiotherapeutics, part 1: effects of solvent on the degradation of radiohalogenation precursors by ${ }^{211}$ At $\alpha$-particles. J Nucl Med. 2005;46:700-706.

14. Pozzi OR, Zalutsky MR. Radiopharmaceutical chemistry of targeted radiotherapeutics, part 2: radiolytic effects of ${ }^{211} \mathrm{At} \alpha$-particles influence $N$-succinimidyl 3-211 At-astatobenzoate synthesis. J Nucl Med. 2005;46:1393-1400.

15. Visser GWM. Inorganic astatine chemistry part II: the chameleon behavior and electrophilicity of At-species. Radiochimica Acta. 1983;33:97-103.

16. Zalutsky MR, Narula AS. A method for the radiohalogenation of proteins resulting in decreased thyroid uptake of radioiodine. Int J Rad Appl Instrum [A]. 1987;38:1051-1055.

17. Garg PK, Archer GE Jr, Bigner DD, Zalutsky MR. Synthesis of radioiodinated $\mathrm{N}$-succinimidyl iodobenzoate: optimization for use in antibody labelling. Int $J$ Rad Appl Instrum [A]. 1989;40:485-490.

18. Larsen RH, Wieland BW, Zalutsky MR. Evaluation of an internal cyclotron target for the production of astatine-211 via the ${ }^{209} \mathrm{Bi}(\alpha, 2 \mathrm{n})^{211}$ At reaction. Appl Radiat Isot. 1996;47:135-143.

19. Koziorowski J, Lebeda O, Weinreich R. A cryotrap as flow reactor for synthesis of ${ }^{211}$ At labelled compounds. Appl Radiat Isot. 1999;50:527-529.

20. Lindegren S, Bäck T, Jensen H. Dry-distillation of astatine-211 from irradiated bismuth targets: a time-saving procedure with high recovery yields. Appl Radiat Isot. 2001;55:157-160.

21. Weber DA, Eckerman KF, Dillman LT, Ryman JC. MIRD: Radionuclide Data and Decay Schemes. New York, NY: Society of Nuclear Medicine; 1989:406415.

22. Wilbur DS. Overcoming the obstacles to clinical evaluation of ${ }^{211}$ At-labeled radiopharmaceuticals. J Nucl Med. 2001;42:1516-1518.

23. Zalutsky MR. Radionuclide therapy. In: Roesch F, ed. Handbook of Nuclear Chemistry Volume 4: Radiochemistry and Radiopharmaceutical Chemistry in Life Sciences. Dordrecht, The Netherlands: Kluwer Academic; 2003:315-348.

24. Khan A. Theoretical studies of the complexes of iodine with methanol, ethanol and acetone. J Chem Phys. 1992;96:1194-1198.

25. Green NJB, Harris R. Spur overlap effects in radiation chemistry. Chem Phys Lett. 1992;198:81-88.

26. Spink JW, Woods RJ. Introduction to Radiation Chemistry. 2nd ed. New York, NY: Wiley; 1976:60-265.

27. Burton M, Magee JL. Advances in Radiation Chemistry Volume 1. New York, NY: Wiley Interscience; 1969:93.

28. Baxendale JH, Wardman P. The Radiolysis of Methanol: Product Yields, Rate Constants, and Spectroscopic Parameters of Intermediates. Washington, DC: National Bureau of Standards; 1975:1-26. Report NSRDS-NBS 54.

29. Zalutsky MR, Narula AS. Astatination of proteins using an N-succinimidyl tri-nbutylstannyl benzoate intermediate. Appl Radiat Isot. 1988;39:227-232.

30. Narula AS, Zalutsky MR. No-carrier-added astatination of $N$-succinimidyl 3-(trin-butylstannyl) benzoate via electrophilic destannylation. Radiochim Acta. 1989; 47:131-135.

31. Zalutsky MR, Garg PK, Friedman HS, Bigner DD. Labeling monoclonal antibodies and $\mathrm{F}\left(\mathrm{ab}^{\prime}\right)_{2}$ fragments with the alpha particle emitting nuclide astatine-211: preservation of immunoreactivity and in vivo localizing capacity. Proc Natl Acad Sci U S A. 1989;86:7149-7153.

32. Talanov VS, Yordanov AT, Garmestani K, et al. Preparation and in vivo evaluation of novel linkers for ${ }^{211}$ At labeling of proteins. Nucl Med Biol. 2004;31: 1061-1071. 